\title{
THE NUMBER OF COUNTS OF BETA-RAYS DETECTED BY AN END WINDOW GEIGER-MUELLER COUNTER (PART II)*
}

\author{
NOBUO KatAYAMA \\ Institute of Earth Science, College of General Education, \\ University of Tokyo
}

\section{Abstract}

The formulae for the number of counts per minute of beta-rays ejected from homogeneous rocks or ores and detected by an end-window Geiger-Mueller counter at a distance more than four centimetres have been calculated with the results as follows:

$$
T=\frac{60 \pi p N W}{C^{*}} G
$$

where

$$
\begin{aligned}
& G=e^{-\frac{\mu_{\mathrm{A}}^{*} H}{[\cos \delta]}} g, \\
& g=\left(\tan ^{-1} \frac{H^{2}+x_{1}^{2}}{X H}-\tan -1 \frac{H}{X}\right) \frac{X H}{4},
\end{aligned}
$$

or

$$
g=\left(\frac{1}{H^{2}}-\frac{1}{H^{2}+X_{1}^{2}}\right) S_{0} H^{4},
$$

and $\mu_{\mathrm{A}}^{*}$ : the absorption coefficient for air regarding the number of betarays $\left(\mathrm{cm}^{-1}\right)$,

$[\cos \delta]$ : the mean velue of $\cos \delta$,

$S_{0}$ : the effective solid angle at the point in which the extension of the central line of Geiger-Mueller tube meets the surface of the object,

other notations being the same as in the previous report.

* Read at the Annual Meeting of the Mineralogical Society of Japan held in Tokyo on June 3, 1955. 


\section{Introduction}

In his previous report ${ }^{1)}$ the writer introduced a formula for calculating the number of counts per munite of beta-rays detected by an end-window Geiger-Mueller counter as follows:

$$
T=\frac{60 \pi p N W}{C^{*}} \sum_{n} G(n, n+1)
$$

where $p:$ the content of a beta-radioactive isotope (in decimal),

$n$ : the number of beta-rays emitted by the beta-radioactive isotore per gram per second,

$W$ : a term representing the absorption effect due to the substaces between the sample and the sensitive volume of the counter,

$C^{*}$ : the mass absorption coefficient for any substance regarding the number of the keta-rays $\left(\mathrm{cm}^{2} \mathrm{gr}^{-1}\right)$,

and $\Sigma G_{(n, n+1)}$ : calculated from the geometrical relation of apparatus and procedure as well as the absorption coefficient of the beta-ray for the sample.

The calculated values are in good agreement with the results of measurement when the distance between the counter and the sample is short.

As we have to measure in the field with a rather long distance between the counter and the object, and as the distance we prefer usually is about ten centimetres owing to the roughness of surface and the heterogeneity of the object, the writer has tried to extend calculation with the view that the results may adequately represent the actual counting.

\section{Effective solid angle}

Denote by $S$ the effective solid angle, that is, the solid angle of the cone, the vertex of which is the point source of beta-ray and the base the basal section of the sensitive volume of the counter, 


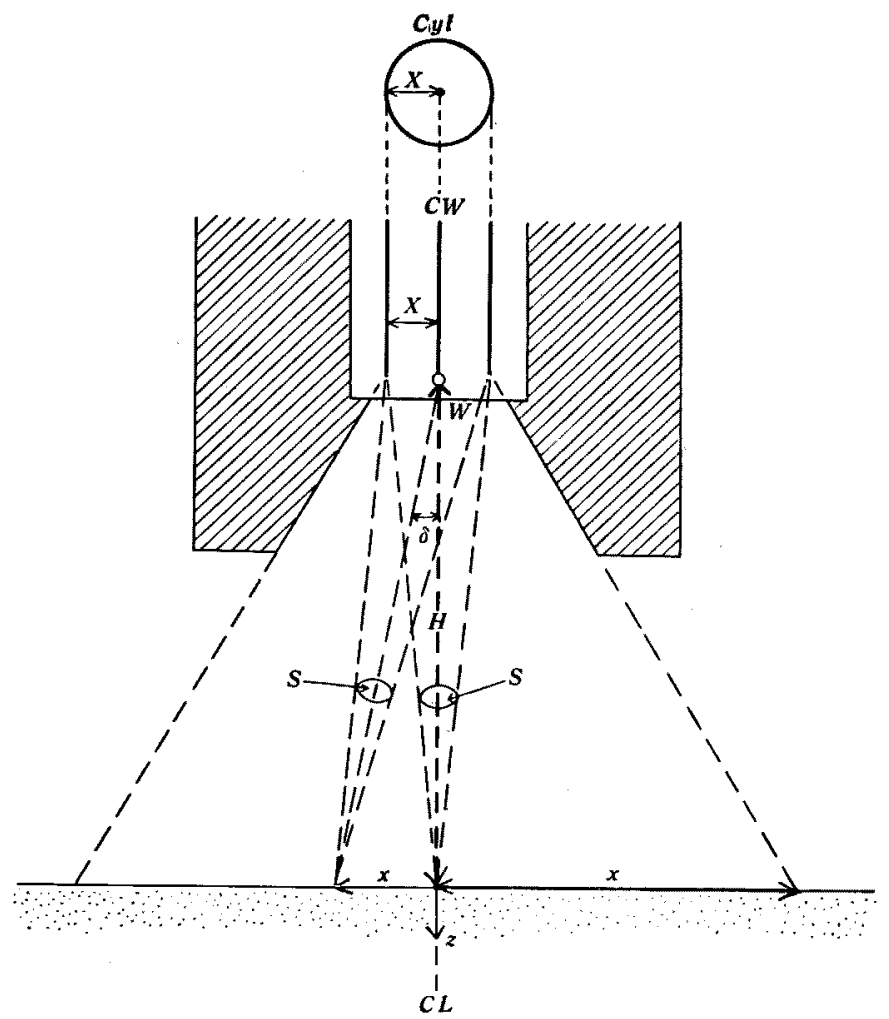

Fig. 5. Scheme of measurement of beta-rays in the field.

Cyl : cylinder cathode. CW : central wire.

W: window, CL: central line.

as designated in the previous paper' (Fig. 5). Since the value of $H$, the distance between the end point of central wire and the surface of the object exceeds by far the value of $X$, the radius of the basal section of the sensitive volume, in this case, the effective solid angle may be expressed approximately as follows:

$$
S \doteqdot \frac{\pi X \cdot X \cos \delta}{4 \pi\left(H^{2}+x^{2}+X^{2} \cos ^{2} \delta\right)}
$$

Substituting $\cos \delta$ by $\frac{H}{\sqrt{H^{2}+x^{2}}}$, we get 


$$
S \doteqdot \frac{X^{3} H \sqrt{H^{2}+x^{2}}}{4\left\{\left(H^{2}+x^{2}\right)^{2}+X^{2} H^{2}\right\}} \text {. }
$$

If the value of $H$ is large enough, $S$ may be expressed as follows:

$$
S \doteqdot \frac{\pi X \cdot X \cos \delta}{4 \pi \frac{H^{2}}{\cos ^{2} \delta}}=S_{0} \cos ^{3} \delta,
$$

where $S_{0}$ denotes the effective solid angle at the point in which the extension of the central line of Geiger-Mueller tube meets the surface of the object, and is given by

$$
S_{0} \doteqdot \frac{\pi X^{2}}{4 \pi H^{2}}=\frac{X^{2}}{4 H^{2}}
$$

\section{Number of counts of beta-rays detected}

Since the solid angle is rather small, the mean declination of tracks of beta-ray, $D$, may be substituted by $\delta$ for approximation. Then, assigning a suitable value to $z_{\llcorner}$so that $S$ and $\cos \delta$ can be regarded practically as constant between $z=0$ and $z_{1}$, equality (9) in the previous paper ${ }^{1)}$ becomes,

$$
d U_{z}=2 \pi x d x \frac{p N}{C^{*}} S \cos \delta\left(1-e^{-\frac{\mu^{*} z_{1}}{\cos \delta}}\right),
$$

where $d U_{z}$ denotes the differential of the number of effective betarays per second ejected from the layer between $z=0$ and $z_{1}$, and $\mu^{*}$, the absorption coefficient for the substance to be measured regarding the number of the beta-rays.

In the previous calculation the absorption of beta-rays by the air between the counter and the sample was neglected, because we treated exclusively of the case with rather small values of $H$. But, in the field we cannot neglect the absorption by air. Hence, introducing the absorption term for air, and applying equalities (21) and (24), $G$ in equality (15) is given by 


$$
G=\frac{1}{2} \int_{0}^{x_{1}} \frac{X^{2} H^{2}}{\left(H^{2}+x^{2}\right)^{2}+X^{2} H^{2}}\left[1-e^{-\frac{\mu^{*} z_{1}}{\cos \delta}}\right] e^{-\frac{\mu_{A}^{*} H}{\cos \delta}} x d x
$$

where $\mu_{A}^{*}$ : absorption coefficient for air regarding the number of beta-rays $\left(\mathrm{cm}^{-1}\right)$.

Since the values of absorption terms do not vary so much within the limitting values of $x$, they may be regarded as a constant which is represented by the mean value of $\cos \delta$ given by

$$
[\cos \delta]=\frac{\int_{0}^{x_{1}} \frac{H}{\sqrt{H^{2}+x^{2}} \cdot 2 \pi x d x}}{\pi x_{1}^{2}}=\frac{2 H\left(\sqrt{H^{2}+x_{1}^{2}}-H\right)}{x_{1}^{2}} .
$$

Hence, equality (25) becomes,

$$
G=e^{-\frac{\mu_{A}^{*} H}{[\cos \delta]}}\left(1-e^{-\frac{\mu^{*} z 1}{[\cos \delta]}}\right)\left(\tan ^{-1} \frac{H^{2}+x^{2}}{X H}-\tan ^{-1} \frac{H}{X}\right) \frac{X H}{4} .
$$

If the value of $H$ is large enough, using equality (22) instead of (21) we get

$$
G=2 \int_{0}^{x_{1}} S_{0} \cos ^{4} \delta\left(1-e^{-\frac{\mu^{*} z_{1}}{[\cos \delta]}}\right) e^{-\frac{\mu_{A H}^{*}}{[\cos \delta]}} x d x
$$

Substituting $\cos \delta$ by $\frac{H}{\sqrt{H^{2}+x^{2}}}$, we get

$$
G=e^{-\frac{\mu_{A}^{*} H}{[\cos \delta 1}}\left(1-e^{-\frac{\mu^{*} z_{1}}{[\cos \delta]}}\right)\left(\frac{1}{H^{2}}-\frac{1}{H^{2}+x_{1}^{2}}\right) S_{0} H^{4}
$$

\section{Numerical calculations for rocks and ores}

As evident from the Table 2 in the previous paper ${ }^{1)}$, the increase of $G$ with the thickness of rocks and substances with comparable density becomes stationary by the layer thinner than four millimetres. In the case the value of $H$ is larger than four centimetres, $S$ and $\cos \delta$ being regarded as constant between $z=0$ and four millimetres, $G$ is given by 


$$
G=e^{-\frac{\mu_{A}^{*} H}{[\cos \delta]}} \mathrm{g}
$$

where

$$
g=\left(\tan ^{-1} \frac{H^{2}+x_{1}^{2}}{X H}-\tan ^{-1} \frac{H}{X}\right) \frac{X H}{4},
$$

or

$$
g=\left(\frac{1}{H^{2}}-\frac{1}{H^{2}+x_{1}^{2}}\right) S_{0} H^{4}
$$

The numerical values of $g$ are calculated for the cases $H=4.5 \mathrm{~cm}$, $5.5 \mathrm{~cm}$., $10 \mathrm{~cm}$. and $100 \mathrm{~cm}$. $X=1 \mathrm{~cm}$., and $x_{1}=1+0.6 \mathrm{Hcm}$. for every case. These values were assigned in accordance with the apparatus and procedure designed by the present author ${ }^{3}$ (Fig. 5). The results are shown in Table 3 .

Table 3. Calculated value of $g$.

\begin{tabular}{l|llll}
\hline$H(\mathrm{~cm})$. & 4.5 & 5.5 & 10 & 100 \\
\hline$g$ & 0.098 & 0.092 & 0.081 & 0.068 \\
\hline
\end{tabular}

Regarding the beta-ray of $\mathrm{K}^{40}$, the value of $C^{*}$ having been determined as $9.1^{1}$, we may assume, for the time being, the value of $\mu_{A}^{*}$ to be 0.0109 , assigning for $\rho^{*}$ the normal density of air. As for the beta-rays emitted from isotopes belonging to the uranium family or to the thorium family, the values of $\mu_{A}^{*}$ were estimated with reference to their usual absorption and the value of $\mu_{A}^{*}$ for $\mathrm{K}^{40}$. Thus the numerical value of $G$ for each beta-radioactive isotope was calculated according to equality (30).

In the expression for $T$ in equality (15), $p N i$ s a constant for each family, if its members are in radioactive equilibrium with each other. In such cases, the particular term for each isotope is $\frac{W}{C^{*}}$ $G$, of which $C^{*}$ can be estimated in the same way as $\mu_{A}^{*}$, and $W$ can be calculated as $e^{-\frac{C^{*} \sigma}{\left[\cos _{\delta}\right]}}$ which expresses the absorption effect of the mica window'). 
The numerical value of $\frac{W}{C^{*}} G$ for each beta-radioactive isotope is tabulated in Table 4. UZ, $\mathrm{RaC}^{\prime \prime}$ anb $\mathrm{AcC}$ are omitted because their beta-radioactivity is small among the uranium family. $\mathrm{RaD}$ and $\mathrm{MsTh}_{1}$ too, are omitted because the energy of their beta-rays is too weak to be detected at such a distance as indicated in Table 4.

Table 4. The numerical value of $\frac{W}{C^{*}} G$ for beta-rays emitted from natural radioactive isotopes.

\begin{tabular}{|c|c|c|c|c|c|c|}
\hline \multicolumn{3}{|c|}{$H(\mathrm{~cm})}$. & 4.5 & 5.5 & 10 & 100 \\
\hline \multicolumn{3}{|c|}{$\mathrm{K}^{40}$} & $9.6 \times 10^{-3}$ & $9.0 \times 10^{-3}$ & $7.4 \times 10^{-3}$ & $2.1 \times 10^{-3}$ \\
\hline \multirow{5}{*}{ 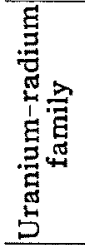 } & Th234 & $\left(U_{1}\right)$ & $8.5 \times 10^{-5}$ & $6.5 \times 10^{-5}$ & $2.4 \times 10^{-5}$ & - \\
\hline & $\mathrm{Pa} 234$ & $\left(\mathrm{UX}_{2}\right)$ & 1. $76 \times 10^{-2}$ & $1.64 \times 10^{-2}$ & $1.41 \times 10^{-2}$ & $6.2 \times 10^{-3}$ \\
\hline & $\mathrm{Pb} 214$ & $(\mathrm{RaB})$ & $2.8 \times 10^{-3}$ & $2.5 \times 10^{-3}$ & $1.9 \times 10^{-3}$ & $9.5 \times 10^{-5}$ \\
\hline & $\mathrm{Bi} 214$ & $(\mathrm{RaC})$ & $2.30 \times 10^{-2}$ & $2.15 \times 10^{-2}$ & $1.85 \times 10^{-2}$ & $9.7 \times 10^{-3}$ \\
\hline & $\mathrm{Bi} 210$ & (RaE) & $7.6 \times 10^{-3}$ & $7.1 \times 10^{-3}$ & $5.8 \times 10^{-3}$ & $1.2 \times 10^{-3}$ \\
\hline \multirow{4}{*}{ 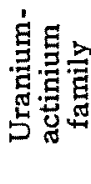 } & Th231 & (UY) & $1.5 \times 10^{-4}$ & 8. $3 \times 10^{-5}$ & $3.1 \times 10^{-5}$ & $\longrightarrow$ \\
\hline & $A c^{227}$ & (Ac) & $1.9 \times 10^{-4}$ & $1.6 \times 10^{-4}$ & $6.6 \times 10^{-5}$ & - \\
\hline & $\mathrm{Pb}^{211}$ & $(\mathrm{AcB})$ & $5.4 \times 10^{-3}$ & $5.0 \times 10^{-3}$ & $4.0 \times 10^{-3}$ & $5.7 \times 10^{-4}$ \\
\hline & $\mathrm{Tl}^{207}$ & $\left(A c C^{\prime \prime}\right)$ & $1.00 \times 10^{-2}$ & $9.3 \times 10^{-3}$ & $7.9 \times 10^{-3}$ & $2.3 \times 10^{-3}$ \\
\hline \multirow{4}{*}{ 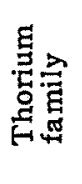 } & $\mathrm{Ac}^{228}$ & $\left(\mathrm{MsTh}_{2}\right)$ & $1.05 \times 10^{-2}$ & $9.8 \times 10^{-3}$ & $8.3 \times 10^{-3}$ & $2.6 \times 10^{-3}$ \\
\hline & $\mathrm{Pb} 212$ & $(T h B)$ & $7.8 \times 10^{-4}$ & $6.7 \times 10^{-4}$ & $4.3 \times 10^{-4}$ & - \\
\hline & $\mathrm{Bi} 212$ & (ThC) & $1.72 \times 10^{-2}$ & $1.61 \times 10^{-2}$ & $1.38 \times 10^{-2}$ & $6.1 \times 10^{-3}$ \\
\hline & $\mathrm{T}^{208}$ & $\left(\mathrm{ThC}^{\prime \prime}\right)$ & $1.30 \times 10^{-2}$ & $1.21 \times 10^{-2}$ & $1.02 \times 10^{-2}$ & $3.8 \times 10^{-3}$ \\
\hline
\end{tabular}

The counts per minute of beta-rays detected for any sample containing one per cent of potassium homogeneously, can be calculated according to equality (15), assigning $p_{\mathrm{K}^{40}}=1.2 \times 10^{-6}$ and $N_{\mathrm{K}^{40}}$ $=2.41 \times 10^{5}$ and the values for $\frac{W}{C^{*}} G$ as in Table 4 .

As for the counts rezarding the uranium family, the calculation formula derived from equality (15) is as follows; 


$$
\begin{aligned}
T_{\mathrm{U}}=60 \pi & \left\{p_{\mathrm{U}^{233}} N_{\mathrm{U}}^{233} \sum_{\mathrm{UX}_{1}, \cdots \operatorname{RaE}}\left[\frac{W}{C^{*}} G\right]\right. \\
& \left.+p_{\mathrm{U}} 235 N_{\mathrm{U}^{235}} \sum_{\mathrm{UY}, \cdots \mathrm{ACC}^{\prime}}\left[\frac{W}{C^{*}} G\right]\right\},
\end{aligned}
$$

where $T_{\mathrm{U}}$ denotes the counts per minute of beta-rays detected for any sample containing one per cent of natural uranium, and $p_{\mathrm{U}} 238$ $=0.00993, p_{\mathrm{U}}^{235}=0.000071, N_{\mathrm{U}^{238}}=1.24 \times 10^{4}$ and $N_{\mathrm{U}^{235}}=8.0 \times 10^{4}$.

Similarly, the calculation formula for the counts regarding the thorium family is given by

$$
\begin{aligned}
& T_{\mathrm{Th}}=60 \pi p_{\mathrm{Th}} N_{\mathrm{Th}}\left\{\left[\frac{W}{C^{*}} G\right]_{\mathrm{MsTh}_{2}}+\left[\frac{W}{C^{*}} G\right]_{\mathrm{ThB}}\right. \\
&\left.+0.65\left[\frac{W}{C^{*}} G\right]_{\mathrm{ThC}}+0.35\left[\frac{W}{C^{*}} G\right]_{\mathrm{ThC}}\right\},
\end{aligned}
$$

where $p_{\mathrm{Th}}=0.01, N_{\mathrm{Th}}=4.13 \times 10^{3}$.

As a matter of course, in equalities (32) and (33) it is assumed

\begin{tabular}{|c|c|c|c|c|c|c|c|}
\hline \multirow[t]{2}{*}{$H(\mathrm{~cm})}$. & \multicolumn{2}{|c|}{4.5} & \multicolumn{2}{|c|}{5.5} & \multirow{2}{*}{$\begin{array}{c}10 \\
{ }_{10}^{10} T\end{array}$} & \multicolumn{2}{|c|}{100} \\
\hline & $4.5 T$ & ${ }_{4.5} T / 10 T$ & ${ }_{5.5} T$ & $5.5 T / 10 T$ & & ${ }_{100} T$ & ${ }_{100} T /{ }_{10} T$ \\
\hline per $1 \% \mathrm{~K}$ & 0.52 & 1.29 & 0.49 & 1.21 & 0.404 & 0.116 & 0.29 \\
\hline per $1 \% \quad \mathrm{U}$ & 1,200 & 1.26 & 1,110 & 1.17 & 950 & 450 & 0.47 \\
\hline per $1 \% \mathrm{Th}$ & 209 & 1.26 & 196 & 1.18 & 166 & 97 & 0.58 \\
\hline
\end{tabular}
that the radioactive equilibrium is held in the sample.

Numerical values thus obtained are tabulated in Table 5.

Table 5. Calculated counts per minute of beta-rays.

\section{Test measurement}

Since it is difficult for measurement in the field to keep such strictly controled conditions as in the laboratory, and since we cannot eliminate the effect of gamma-rays, $T_{m}$, the number of counts per minute measured in the field somewhat differs from the calculated value of $\boldsymbol{T}$. For a definite apparatus and a definite beta-active iso- 
tope, the ratio of $T_{m}$ to $T$ may be considered as a constant $k$, that is

$$
T_{m}=k T \text {. }
$$

Hence, the ratio between $T_{m}$ 's measured at different distance $H$, may be equal to the ratio between the relevant $T$ 's calculated.

For the first example, measuring the radioactivity of chemical pure $\mathrm{K}_{2} \mathrm{SO}_{4}$ at $H=0.8 \mathrm{~cm}$., $1.0 \mathrm{~cm} ., 4.5 \mathrm{~cm}$. and $5.5 \mathrm{~cm}$., we obtained the ratios as tabulated in Table 6 . In the case at $H=0.8 \mathrm{~cm}$. and $1.0 \mathrm{~cm}$., $x_{1}$ was $1.0 \mathrm{~cm}$. and $\rho^{*}=1.14$, and the calculated value $T$ was obtained from Table 2 in the previous paper ${ }^{1}$.

Table 6. Measured and calculated ratio between the counts for $\mathrm{K}_{2} \mathrm{SO}_{4}$ at different distances.

\begin{tabular}{l|ccc}
\hline & $0.8 T: 1.0 T$ & $1.0 T: 4.5 T$ & $4.5 T:{ }_{5.5} T$ \\
\hline Measured value & 1.21 & 0.94 & 1.04 \\
Calculated value & 1.24 & 1.03 & 1.07 \\
\hline
\end{tabular}

Table 7. Measured and calculated ratios between the counts for a granite at different distances.

\begin{tabular}{l|c}
\hline & $5.5 T:{ }_{10} T$ \\
\hline Measured value & 1.15 \\
Calculated value & $1.17 \sim 1.21$ \\
\hline
\end{tabular}

For the second example, a ground granite block was measured at $H=5.5 \mathrm{~cm}$. and $10 \mathrm{~cm}$.. At these distances the distribution of radio-active source in the granite may be regarded as homogeneous, so the calculation above described may be applied in this case as well. The results are shown in Table 7.

\section{Discussion}

Throughout these measurements the apparatus used was Aloka DC-P13) made by the Electro-Medical Research Institute Ltd.. This apparatus was designed by the present author in order to measure the beta-radioactivity of ores and rocks in the field as precisely as possible, and to enable $u_{s}$ to estimate the content of racioactive 
isotopes in them. The calculations described in this paper are performed in order to give a foundation for that estimation. The validity of the calculation is confirmed by the results of measurement shown in Tables 6 and 7, allowing fhe error less than ten per cent.

Considering the roughness of the surface of ores and rocks as well as their heterogeneity, the author assignes the value of $H$ as $10 \mathrm{~cm}$. for usual measurements. With this value of $H$, the radioactivity of the air between the sample and the counter is negligible in ordinary case but, in the field rich in radioactive minerals, especially in the gallery, it cannot be neglected. This problem will be discussed in another paper ${ }^{2}$.

\section{Acknowledgement}

The writer wishes to express his gratitude to Professor T. Ito, Institute of Mineralogy, University of Tokyo, for critically reading the manuscript. Thanks are also due to the Geological Survey of Japan for furnishing the measuring apparatus, and to the ElectroMedical Research Institute Ltd. for collaborating with the writer in constructing the apparatus.

This investigation was aided by a grant from the Scientific Research Fund of the Department of the Education.

\section{REFERENCES}

1) Katayama, N.: Miner. Jour., 1, 170 (1954).

2) Katayama, N.: Szi. Pap. Coll. Gan. Ed. Univ. Tokyo, 5, No. 2 (1955).

3) Katayamı, N.: to be pu'blis.zed in Mining Gzol., 6, (1956), in Japanese.

Manuscript received August 20, 1955. 